\title{
BMJ Open Prognostic factors of inhospital death in elderly patients: a time-to-event analysis of a cohort study in Martinique (French West Indies)
}

\author{
Lidvine Godaert, ${ }^{1}$ Seendy Bartholet, ${ }^{1}$ Fréderique Dorléans, ${ }^{2}$ Fatiha Najioullah, ${ }^{3}$ \\ Sebastien Colas, ${ }^{1}$ Jean-Luc Fanon, ${ }^{1}$ André Cabié, ${ }^{4}$ Raymond Césaire, ${ }^{3}$ \\ Moustapha Dramé ${ }^{5,6}$
}

To cite: Godaert L, Bartholet S, Dorléans F, et al. Prognostic factors of inhospital death in elderly patients: a timeto-event analysis of a cohort study in Martinique (French West Indies). BMJ Open 2018;8:e018838. doi:10.1136/ bmjopen-2017-018838

- Prepublication history for this paper is available online. To view these files, please visit the journal online (http://dx.doi. org/10.1136/bmjopen-2017018838).

Received 26 July 2017 Revised 29 November 2017 Accepted 30 November 2017

Check for updates

${ }^{1}$ Department of Geriatrics, University Hospitals of Martinique, Martinique, France ${ }^{2}$ Inter-regional Epidemiology Unit (CIRE), Regional Health Agency (ARS) of Martinique, Martinique, France

${ }^{3}$ Department of Virology, University Hospitals of Martinique, Martinique, France ${ }^{4}$ Department of Infectious Diseases, University Hospitals of Martinique, Martinique, France ${ }^{5}$ Faculty of Medicine, University of Reims Champagne-Ardenne, Reims, France

${ }^{6}$ Department of Research and Public Health, University Hospital of Reims, Robert Debré Hospital, Reims, France

Correspondence to

Dr Lidvine Godaert;

lidvinegodaert@me.com

\section{ABSTRACT}

Objective The primary objective was to identify predictive factors of inhospital death in a population of patients aged 65 years or older hospitalised with Chikungunya virus (CHIKV) infection. The secondary aim was to develop and validate a predictive score for inhospital death based on the predictors identified.

Design Longitudinal retrospective study from January to December 2014.

Setting University Hospital of Martinique.

Participants Patients aged $\geq 65$ years, admitted to any clinical ward and who underwent reverse transcription PCR testing for CHIKV infection.

Outcome Independent predictors of inhospital death were identified using multivariable Cox regression modelling. A predictive score was created using the adjusted HRs of factors associated with inhospital death. Receiver operating characteristic curve analysis was used to determine the best cut-off value. Bootstrap analysis was used to evaluate internal validity.

Results Overall, 385 patients aged $\geq 65$ years were included (average age: $80 \pm 8$ years). Half were women, and 35 (9.1\%) died during the hospital stay. Seven variables were found to be independently associated with inhospital death (concurrent cardiovascular disorders: HR $11.8,95 \% \mathrm{Cl} 4.5$ to 30.8 ; concurrent respiratory infection: $\mathrm{HR} 9.6,95 \% \mathrm{Cl} 3.4$ to 27.2 ; concurrent sensorimotor deficit: $\mathrm{HR} 7.6,95 \% \mathrm{Cl} 2.0$ to 28.5 ; absence of musculoskeletal pain: $\mathrm{HR} 2.6,95 \% \mathrm{Cl} 1.3$ to 5.3 ; history of alcoholism: HR $2.5,95 \% \mathrm{Cl} 1.1$ to 5.9 ; concurrent digestive symptoms: HR 2.4, 95\% $\mathrm{Cl} 1.2$ to 4.9 ; presence of confusion or delirium: $\mathrm{HR} 2.1,95 \% \mathrm{Cl} 1.1$ to 4.2 ). The score ranged from 0 to 25 , with an average of $6 \pm 6$. The area under the curve was excellent $(0.90 ; 95 \% \mathrm{Cl} 0.86$ to 0.94$)$. The best cut-off value was a score $\geq 8$ points, with a sensitivity of $91 \%$ (82\%-100\%) and specificity of $75 \%(70 \%-80 \%)$.

Conclusions Signs observed by the clinician during the initial examination could predict inhospital death. The score will be helpful for early management of elderly subjects presenting within 7 days of symptom onset in the context of CHIKV outbreaks.

\section{INTRODUCTION}

Chikungunya virus (CHIKV) infection is an emergent arthropod-borne alpha-virus
Strengths and limitations of this study

- This is the first study to identify independent prognostic factors of inhospital death in a population of elderly subjects infected with Chikungunya virus.

- The sample size is substantial.

- The diagnosis of infection was confirmed in all patients by reverse transcription PCR.

- Our findings warrant external validation.

transmitted by Aedes mosquito bites and causes fever with debilitating arthritic illness. ${ }^{1} 2$ CHIKV infection has spread following an endemic-epidemic pattern in Asia, the Indian Ocean Islands and more recently in the French West Indies. ${ }^{1}$ Prevalence is high in immunologically naive people, ${ }^{3-5}$ and millions of persons have been affected to date. ${ }^{6}$ Seroepidemiological surveys in blood donors identified a final seroprevalence of $48.1 \%$ in Guadeloupe and $41.9 \%$ in Martinique. $^{7}$ The mortality and morbidity related to this infection are poorly documented. Some authors have reported excess lethality $^{8-14}$ or excess mortality ${ }^{15}$ during epidemics, or both, ${ }^{17}$ with reported rates ranging from $0.1 \%$ to $48 \%$ according to the study population, location and methods of assessment. Most published findings concern young adults, ${ }^{8151819}$ and although certain authors have suggested that age, comorbidity and alcohol consumption are prognostic factors of death, ${ }^{111819}$ mortality and morbidity data are scarce for subjects aged 65 years and over $(65+)$.

Therefore, our primary objective was to identify prognostic factors of inhospital death in a population of patients aged $65+$ hospitalised with CHIKV infection. The secondary objective was to develop 
a predictive score for inhospital death based on the predictors identified.

\section{METHODS}

\section{Study design and subjects}

This cohort study was performed in the University Hospital of Martinique (French West Indies) from retrospective cases. Eligible patients were aged 65 years or older, admitted to any clinical ward (including intensive care units (ICUs) and all specialised units) from 10 January to 31 December 2014 and who underwent biological testing using reverse transcription PCR (RT-PCR) for CHIKV infection. Only patients presenting within 7 days of the onset of symptoms were included. There was no concurrent circulation of dengue at the time of the inclusions.

\section{Data collection}

Data were retrospectively collected from medical charts. We recorded baseline characteristics, including age and sex, presence or absence of fever and musculoskeletal pain (including arthralgia and myalgia). We also recorded history of diabetes, heart failure or alcoholism and presence or absence of any of the following unusual concurrent clinical manifestations at admission: digestive symptoms (nausea, diarrhoea and abdominal pain), CHIKV-associated central nervous system (CNS) diseases (including confusion or delirium, seizure, syncope, encephalitis and sensorimotor deficit), renal failure, respiratory disorders (including dyspnoea, respiratory infection based on clinical examination, positive sputum Gram stain or culture for bacteria or simply an infiltrate on chest X-ray), cardiovascular disorders (including rhythm disorder, cardiac decompensation and myocardial ischaemia), decompensated diabetes (with negative impact on glycaemic control), dermatological signs (including maculopapular rash, exanthema, erysipelas and purpura) and haematological signs (thrombocytopenia $<100 \times 109 / \mathrm{L}$, thrombocytosis $>400 \times 109 / \mathrm{L}$, leucocytosis $>0.01 \times 10^{9} / \mathrm{L}$ and leucopenia $<0.0015 \times 10^{9} / \mathrm{L}$ ). The comorbidity burden was assessed using Charlson Comorbidity Index. ${ }^{20}$ Charlson Comorbidity Index is a tool that assesses the comorbidity burden for a given individual. It is composed of 19 comorbid conditions including diabetes, with or without complications, congestive heart failure, peripheral vascular disease, chronic pulmonary disease, mild or severe liver disease, hemiplegia, renal disease, leukaemia, lymphoma, solid tumour (with or without metastasis) and AIDS. Each of these conditions is weighted by a coefficient $(1,2,3$ or 6$)$ according to their potential influence on mortality. The higher the score, the higher the comorbidity burden. Exanthema, myalgia, back pain, headache, vomiting and diarrhoea without impact on the overall general health status were considered as signs usually accompanying the typical acute phase course of CHIKV infection, in line with the WHO definition. ${ }^{21}$ All patients included in this study underwent serum sample testing using RT-PCR with the RealStar Chikungunya RT-PCR Kit (Altona Diagnostics, Hamburg, Germany).

\section{Ethical considerations}

The study was performed in accordance with the Declaration of Helsinki and French legislation relating to research involving human beings. According to French law, patients have the right to refuse to participate in studies by notifying their opposition orally.

\section{Statistical analysis}

Quantitative variables are described as mean $\pm \mathrm{SD}$, and categorical variables as number and percentage.

Bivariable relationships between each potential prognostic factor and time to inhospital death were analysed using a Cox model. Variables with a P value of less than 0.2 were entered into the multivariable model. KaplanMeier curves were constructed, and the log-rank test was used to test survival differences between score groups $(<8 \mathrm{vs} \geq 8)$. A multivariable Cox model was constructed to derive the prediction model. Time to inhospital death was the primary outcome. Survival time was defined as the duration between hospital admission and the date of death or date of last contact. The vital status was updated from the hospital wards (using the hospital network data-processing system). Baseline characteristics were considered as potential predictors for inhospital death. A manual stepwise method was used to identify variables independently associated with time to inhospital death, after checking for confounders and interactions. The proportionality of risks over time, which is a condition of validity for the Cox model, was verified graphically. ${ }^{22}$ Results are presented as HR and 95\% CI.

For the score development, a point value was assigned to each independent factor according to the adjusted HR of the Cox model. Point values were rounded to the nearest integer and summed.

The area under the receiver operating characteristic (AUROC) curve was calculated. Bootstrap analysis was also performed to evaluate the internal validity of the model. Replication on 2000 different samples drawn with replacement was performed using the bootstrap method. The ROC curve was used to determine the cut-off value of the score that best discriminated patients with regard to the outcome. The $95 \%$ CI of the AUROC was also obtained using bootstrap methods. The following diagnostic indices and their 95\% CI were computed: sensitivity, specificity, positive predictive value and negative predictive value.

A P value $<0.05$ was considered statistically significant. Statistical analyses were performed using SAS V.9.4 (SAS Institute). The ROC curves were computed using IBM SPSS software release V.21.

\section{RESULTS}

During the epidemic that was ongoing from 10 January to 31 December 2014 in Martinique, 471 patients aged 
Table 1 Baseline characteristics of the study population of 385 elderly patients with Chikungunya virus infection

\begin{tabular}{lrr}
\hline Characteristic & $\mathbf{n}$ & \multicolumn{1}{c}{$\%$} \\
\hline Age $\geq 85$ years & 111 & 28.8 \\
\hline Female & 193 & 50.1 \\
\hline Fever & 331 & 86.0 \\
\hline Musculoskeletal pain & 294 & 76.4 \\
\hline Including arthralgia & 283 & 73.5 \\
\hline Including myalgia & 113 & 29.4 \\
\hline History of diabetes & 145 & 37.7 \\
\hline History of heart failure & 34 & 8.8 \\
\hline History of alcoholism & 36 & 9.4 \\
\hline Unusual concurrent clinical manifestations & & \\
\hline Digestive symptoms & 100 & 26.0 \\
\hline CNS diseases & 180 & 46.8 \\
\hline Including confusion or delirium & 119 & 30.9 \\
\hline Including sensorimotor deficit & 10 & 2.6 \\
\hline Renal failure & 117 & 30.4 \\
\hline Respiratory disorders & 82 & 21.3 \\
\hline Including respiratory infection & 17 & 4.4 \\
\hline Cardiovascular disorders & 97 & 25.2 \\
\hline Decompensated diabetes & 27 & 7.0 \\
\hline Haematological signs & 54 & 14.0 \\
\hline CNS, centralnervous system. & & \\
\hline
\end{tabular}

CNS, central nervous system.

$65+$ were hospitalised with a diagnosis of CHIKV infection confirmed by RT-PCR. Among these, 86 were not included because the time between onset of symptoms and RT-PCR testing exceeded 7 days. Patients who were excluded did not differ significantly from those who were included in terms of age, sex or comorbidity burden. CHIKV RT-PCR results were available a maximum of 24 hours after blood sampling. In total, 385 patients aged 65 years and over were included in this study. Average age was $80 \pm 8$ years; half $(50.1 \%)$ were women. The mean time since symptom onset was 2.4 \pm 1.9 days. The average Charlson Comorbidity Index was $1.3 \pm 1.4$. Other baseline characteristics are presented in table 1.

In all, 35 patients $(9.1 \%)$ died during the hospital stay. Six patients died in the first 24 hours after admission $(16.2 \%)$. Thirty-five patients were admitted to the ICU, of whom $16(45.7 \%)$ died during hospital stay. Median time to inhospital death was 58 days (range: 1-84 days). Median length of stay was 7 days (range: 1-84 days). No statistically significant differences were found between deceased and non-deceased patients with regard to age (81.9 \pm 9.9 vs $80.3 \pm 7.4$, respectively) or Charlson Comorbidity Index (1.6 \pm 1.3 vs $1.3 \pm 1.4$, respectively). As shown in figure 1 , subjects with score $<8$ had significantly higher survival rates than those with score $\geq 8(\mathrm{P}<0.0001)$.

By multivariable analysis, seven independent predictors of inhospital death were identified, namely concurrent

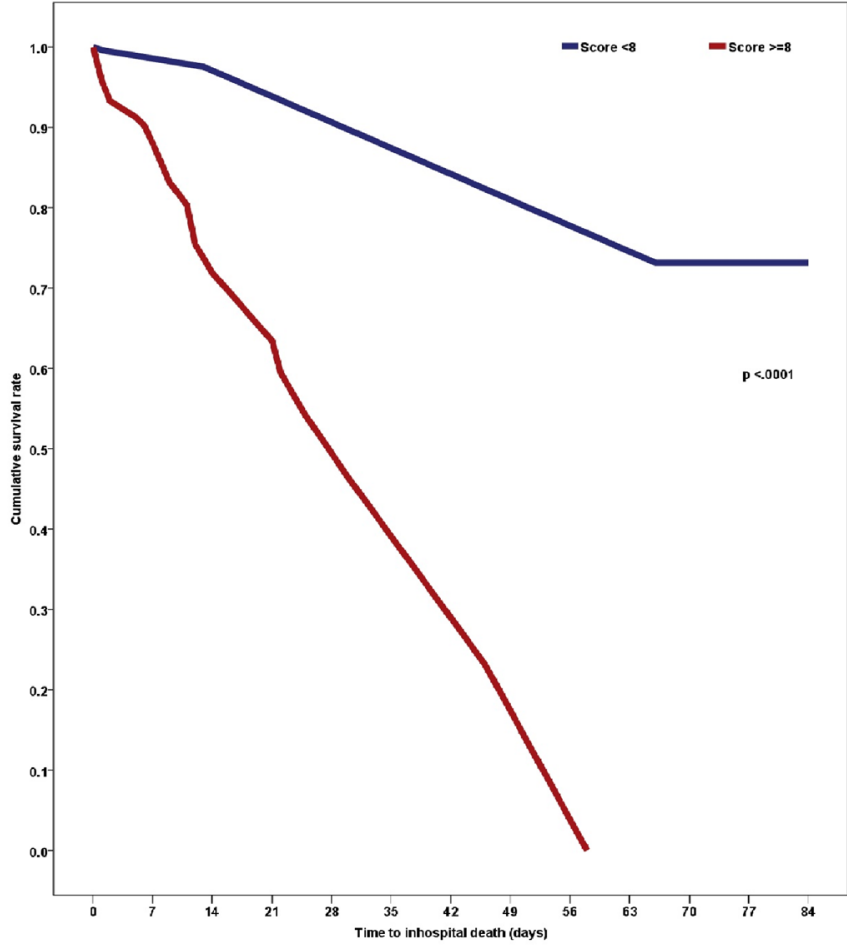

Figure 1 The Kaplan-Meier curves estimate the survival of subjects according to their score groups (score $\geq 8$ vs score $<8$ ). The two curves were compared using the log-rank test.

cardiovascular disorders, concurrent respiratory infection, concurrent sensorimotor deficit, absence of musculoskeletal pain, history of alcoholism, concurrent digestive symptoms and presence of confusion or delirium.

The independent predictors of inhospital death are presented in table 2.

Bootstrap methods showed good internal validity of the final model (data not shown).

The point values assigned to each of the predictors identified in the final model are listed in table 2. The scores observed among the study population ranged from 0 to 25 ; with an average of $6 \pm 6$. Figure 2 presents the ROC curve. The uncorrected AUROC was as satisfactory as the bootstrapped AUROC, namely 0.90 (95\% CI 0.86 to 0.94 ) and 0.90 (95\% CI 0.90 to 0.90 ), respectively. A score of 8 or higher was considered as the cut-off that best identified patients with a lower risk of inhospital death. The diagnostic performances of the score dichotomised at 8 points are presented in table 3 .

\section{DISCUSSION}

In our cohort study, seven prognostic factors were associated with in-hospital death (namely cardiovascular disorders, respiratory infection, sensorimotor deficit, absence of musculoskeletal pain, digestive symptoms and presence of confusion or delirium). In all, $9.1 \%$ of patients aged $65+$ with CHIKV infection died during their hospital stay. This result is similar to those reported by Economopoulou et $a l$ in $2009^{11}$ in an adult population 
Table 2 Independent predictors of inhospital death for elderly patients with CHIKV infection and the corresponding point values of the developed score

\begin{tabular}{|c|c|c|c|c|c|}
\hline Predictors & $\mathrm{HR}^{*}(95 \% \mathrm{Cl})$ & $P$ value & aHR† $(95 \% \mathrm{Cl})$ & $P$ value & Points \\
\hline Age $\geq 85$ years & 1.4 (0.7 to 2.8$)$ & 0.36 & & & \\
\hline Male sex & 1.1 (0.6 to 2.2$)$ & 0.73 & & & \\
\hline Fever & 1.4 (0.5 to 3.6$)$ & 0.51 & & & \\
\hline Arthralgiał & 0.6 (0.3 to 1.1$)$ & 0.10 & & & \\
\hline Concurrent renal failure $\neq$ & 3.5 (1.7 to 7.1$)$ & 0.0007 & & & \\
\hline Concurrent decompensated diabetes & 0.8 (0.2 to 2.5$)$ & 0.66 & & & \\
\hline Concurrent CNS diseases $\ddagger$ & 1.7 (0.8 to 3.3$)$ & 0.16 & & & \\
\hline Including sensorimotor deficitł & 2.8 (0.8 to 9.2$)$ & 0.09 & 7.6 (2.0 to 28.5$)$ & 0.003 & 8 \\
\hline Including confusion or delirium $\ddagger$ & 1.9 (0.9 to 3.6$)$ & 0.07 & 2.1 (1.1 to 4.2$)$ & 0.04 & 2 \\
\hline Concurrent cardiovascular disordersł & $6.0(2.8$ to 12.9$)$ & $<0.0001$ & $11.8(4.5$ to 30.8$)$ & $<0.0001$ & 12 \\
\hline Concurrent respiratory infection $\ddagger$ & 3.1 (1.3 to 7.6$)$ & 0.01 & 9.6 (3.4 to 27.2$)$ & $<0.0001$ & 10 \\
\hline Absence of musculoskeletal pain $\ddagger$ & 2.1 (1.1 to 4.0$)$ & 0.04 & $2.6(1.3$ to 5.3$)$ & 0.009 & 3 \\
\hline History of alcoholism $\ddagger$ & 2.5 (1.1 to 5.7$)$ & 0.03 & 2.5 (1.1 to 5.9$)$ & 0.04 & 3 \\
\hline Concurrent digestive symptomsł & 2.2 (1.1 to 4.3$)$ & 0.02 & 2.4 (1.2 to 4.9$)$ & 0.02 & 2 \\
\hline
\end{tabular}

*Unadjusted HR.

†aHR from the final Cox model.

$\ddagger$ Variables proposed to the multivariable model.

aHR, adjusted HR; CHIKV, Chikungunya virus; CNS, central nervous system.

hospitalised during a CHIKV outbreak in Reunion Island with atypical or severe cases (median age: 70 years (range: 15-95 years)). These authors identified alcohol consumption and age over 85 years as prognostic factors of death. In our cohort study, alcohol consumption was

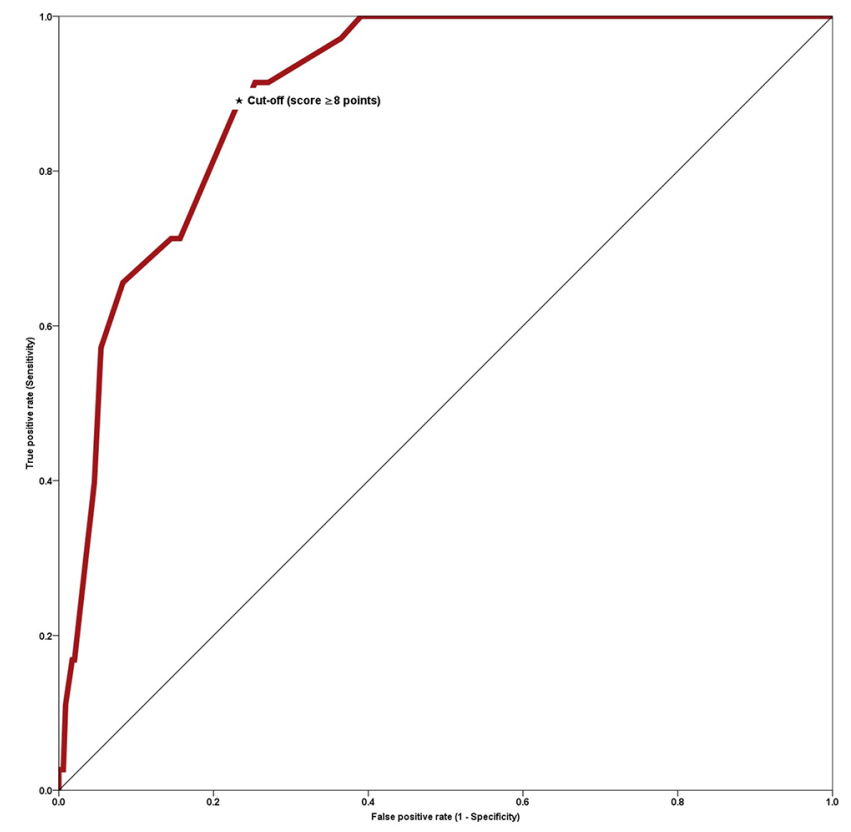

Figure 2 The receiver operating characteristic curve is a plot of the true positive rate (sensitivity) against the false positive rate (1-specificity). The cut-off value represents the point on the curve that maximises both sensitivity and specificity. In this analysis, the cut-off value identified to best distinguish between deceased and non-deceased subjects was 8 points $(\star)$. also associated with inhospital death, but no significant differences were found between deceased and non-deceased patients with regard to age and comorbidity index. However, in the elderly population, we cannot exclude the possibility that previously undetected comorbidities may flare up in the context of an acute infection. ${ }^{23}$ The lack of any difference between those who died and those who survived in terms of comorbidities pleads in favour of a moderate influence of pre-existing disease on the clinical course of CHIKV infection. This is in line with a previous report by Godaert $e t a l^{24}$ about the clinical forms of CHIKV infection in subjects aged 65 years and over during the 2014 outbreak in Martinique. They reported that in almost $70 \%$ of cases, elderly patients presented at the acute phase with either an atypical form (ie, fever and arthralgia and one other clinical sign not considered as typical) or a form that is not included in the WHO classification $^{21}$ (organ dysfunction, absence of fever and

Table 3 Diagnostic performances of the score dichotomised at 8 points ${ }^{*}$ to predict inhospital death in elderly patients with CHIKV infection.

\begin{tabular}{lrl}
\hline & Value & $\mathbf{9 5 \%}$ Cl \\
\hline Sensitivity & $91 \%$ & $82 \%$ to $100 \%$ \\
Specificity & $75 \%$ & $70 \%$ to $80 \%$ \\
\hline Positive predictive value & $26 \%$ & $18 \%$ to $34 \%$ \\
Negative predictive value & $99 \%$ & $98 \%$ to $100 \%$ \\
\hline Correctly predicted rate & $76 \%$ & $72 \%$ to $80 \%$ \\
\hline
\end{tabular}

${ }^{*}$ A score $\geq 8$ indicates a higher risk of inhospital death. $\mathrm{CHIKV}$, Chikungunya virus. 
absence of arthralgia, among others). In the report by Godaert $e t a l,{ }^{24}$ patients aged 65 years or over who had the typical form of CHIKV had fewer comorbidities than aged patients in whom the CHIKV presentation was severe or unclassifiable. In the younger group, only patients with an unclassifiable form had more comorbidities. At a similar level of comorbidities, elderly subjects did not have severe forms more frequently than their younger counterparts. Therefore, it would seem that pre-existing disease cannot alone explain the excess of mortality in the elderly population.

In this cohort study, symptoms present at admission appear to be predictive of the clinical course. WHO recently established definitions for acute, atypical and severe cases of CHIKV infection. ${ }^{21}$ Dysfunction of at least one organ system that is life-threatening and requires hospitalisation defines severe clinical cases of CHIKV infection. In the literature, seroprevalence of CHIKV infection and the risk of hospitalisation increase with age, ${ }^{725}$ and older people are reportedly at greater risk of presenting atypical or severe clinical cases of CHIKV infection. ${ }^{11}{ }^{1724}$ In our cohort study, cardiovascular disorders, respiratory infection, sensorimotor deficit, absence of musculoskeletal pain, digestive symptoms and presence of confusion or delirium were identified as independent predictors of inhospital death. These signs were also observed in patients without known underlying conditions and, therefore, seem to be integral components of the clinical presentation at the acute phase in this population. These signs can be observed by the clinician during the initial examination and appear to be very informative. In patients with a positive diagnosis of CHIKV, the presence of these signs could guide therapeutic management.

Considering all the predictive factors identified by multivariable analysis, we developed a score to predict the risk of inhospital death in older patients with CHIKV infection. It was derived using usual and accurate statistical methods. Moreover, its internal validation was very good. Indeed, the accuracy of our score was excellent (AUROC $=0.90$ ) and remained unchanged between iterations of internal validation using the bootstrap method. During an outbreak, this score could be very helpful since the signs or symptoms that are components of the score are not among the clinical features usually described in CHIKV infection. ${ }^{21}$ The score is easy to calculate by simply summing the different point values corresponding to the seven predictors. A cut-off of less than 8 points identifies patients who are at lower risk of dying during their hospital stay, with excellent discriminant properties.

Patients with CHIKV infection with clinical signs suggestive of likely adverse outcome should receive specialised care from a medical team including a specialist in geriatric medicine. Ellis $e t a l^{26}$ suggested in their meta-analysis that geriatric intervention increases a patient's likelihood of being alive after an emergency admission to hospital. This practice is becoming increasingly common in some medical specialities, such as oncology.
Some limitations of this study deserve to be underlined, and the findings must be interpreted with caution. Our study concerned only hospitalised patients, presenting within 7 days of the onset of symptoms. We do not have data regarding patients admitted more than 7 days after onset of symptoms. All data were collected from medical files. The absence of a documented symptom does not necessarily mean that the patient did not experience this symptom. For instance, the number of CHIKV-associated CNS disease would have been underestimated, leading thus to information bias. All patients had atypical or severe clinical cases of CHIKV infection according to definitions of the expert group of WHO. ${ }^{21}$ Although Godaert et al recently showed that around $70 \%$ of elderly patients present with these clinical forms, we do not have information about mortality in patients presenting with typical acute clinical cases of CHIKV infection. Patients may have received different interventions after admission (ie, ICU admission vs general ward), which may also have affected mortality. The main limitation regarding the score relates to the absence of external validation. However, as outbreaks are rare, as are geriatric care units involved in CHIKV research, it is very challenging to constitute a validation cohort.

A strong point of this study is the fact that it is, to the best of our knowledge, the first study to identify predictors of in-hospital death in a population of elderly subjects infected by CHIKV. Among the other strengths of our study, we can underline the fact that all subjects included underwent RT-PCR serum testing in the same laboratory, using the same commercial kit. Furthermore, the sample size is large.

In conclusion, our results show that CHIKV infection can be fatal in atypical or severe clinical cases. A score based on easy-to-access clinical features obtained in routine practice could identify patients at higher risk of adverse outcomes during the hospital stay. This score could be helpful to guide management and patient supervision in the context of CHIKV outbreaks.

Acknowledgements We thank Fiona Ecarnot (EA3920, University Hospital Besancon, France) for editorial assistance.

Contributors LG made major contributions to the conception and design of the study; contributed to the acquisition, analysis and interpretation of data; drafted the manuscript for important intellectual content and gave final approval of the version to be published. SB contributed to the conception and design of the study and acquisition and interpretation of data; drafted the manuscript for important intellectual content and gave final approval of the version to be published. FD and SC contributed to the acquisition of data; drafted the manuscript for intellectual content and gave final approval of the version to be published. FN, J-LF and RC contributed to the conception and design of the study and interpretation of data; drafted the manuscript for important intellectual content and gave final approval of the version to be published. AC contributed to the conception and design of the study; drafted the manuscript for intellectual content and gave final approval of the version to be published. MD contributed to the conception and design of the study and acquisition, analysis and interpretation of data; drafted the manuscript for important intellectual content and gave final approval of the version to be published. All authors read and approved the final manuscript.

Funding This research received no specific grant from any funding agency in the public, commercial or not-for-profit sectors.

Competing interests None declared. 
Patient consent Obtained.

Ethics approval The study was approved by the French national authority for the protection of privacy and personal data (Commission nationale de l'informatique et des libertés, CNIL).

Provenance and peer review Not commissioned; externally peer reviewed.

Data sharing statement Data are from the Chikungunya study and are not publicly available to protect patient privacy. They can be requested at lidvinemichele.godaert-simon@chu-martinique.fr (Department of Geriatrics, Martinique University Hospital).

Open Access This is an Open Access article distributed in accordance with the Creative Commons Attribution Non Commercial (CC BY-NC 4.0) license, which permits others to distribute, remix, adapt, build upon this work non-commercially, and license their derivative works on different terms, provided the original work is properly cited and the use is non-commercial. See: http://creativecommons.org/ licenses/by-nc/4.0/

(C) Article author(s) (or their employer(s) unless otherwise stated in the text of the article) 2018. All rights reserved. No commercial use is permitted unless otherwise expressly granted.

\section{REFERENCES}

1. Thiberville SD, Moyen N, Dupuis-Maguiraga L, et al. Chikungunya fever: epidemiology, clinical syndrome, pathogenesis and therapy. Antiviral Res 2013;99:345-70.

2. Simon F, Javelle E, Cabie A, et al. French guidelines for the management of chikungunya (acute and persistent presentations). November 2014. Med Mal Infect 2015;45:243-63.

3. Sissoko D, Moendandze A, Malvy D, et al. Seroprevalence and risk factors of chikungunya virus infection in Mayotte, Indian Ocean, 2005-2006: a population-based survey. PLoS One 2008;3:e3066.

4. Gérardin P, Guernier V, Perrau J, et al. Estimating Chikungunya prevalence in La Réunion Island outbreak by serosurveys: two methods for two critical times of the epidemic. BMC Infect Dis 2008;8:99.

5. Staples JE, Breiman RF, Powers AM. Chikungunya fever: an epidemiological review of a re-emerging infectious disease. Clin Infect Dis 2009;49:942-8.

6. Petersen LR, Powers AM. Chikungunya: epidemiology. F1000Res 2016:5.

7. Gallian P, Leparc-Goffart I, Richard P, et al. Epidemiology of Chikungunya Virus Outbreaks in Guadeloupe and Martinique, 2014: An Observational Study in Volunteer Blood Donors. PLoS Negl Trop Dis 2017;11:e0005254.

8. Renault $\mathrm{P}$, Josseran L, Pierre V. Chikungunya-related fatality rates, Mauritius, India, and Reunion Island. Emerg Infect Dis 2008;14:1327a-1327.
9. Labeaud AD, Bashir F, King CH. Measuring the burden of arboviral diseases: the spectrum of morbidity and mortality from four prevalent infections. Popul Health Metr 2011;9:1.

10. Lemant J, Boisson V, Winer A, et al. Serious acute chikungunya virus infection requiring intensive care during the Reunion Island outbreak in 2005-2006. Crit Care Med 2008;36:2536-41.

11. Economopoulou A, Dominguez M, Helynck B, et al. Atypical Chikungunya virus infections: clinical manifestations, mortality and risk factors for severe disease during the 2005-2006 outbreak on Réunion. Epidemiol Infect 2009;137:534-41.

12. Rollé A, Schepers K, Cassadou S, et al. Severe Sepsis and Septic Shock Associated with Chikungunya Virus Infection, Guadeloupe, 2014. Emerg Infect Dis 2016:22:891-4.

13. Crosby L, Perreau C, Madeux B, et al. Severe manifestations of chikungunya virus in critically ill patients during the 2013-2014 Caribbean outbreak. Int J Infect Dis 2016;48:78-80.

14. Tandale BV, Sathe PS, Arankalle VA, et al. Systemic involvements and fatalities during Chikungunya epidemic in India, 2006. J Clin Virol 2009;46:145-9.

15. Mavalankar D, Shastri P, Bandyopadhyay T, et al. Increased mortality rate associated with chikungunya epidemic, Ahmedabad, India. Emerg Infect Dis 2008;14:412-5.

16. Josseran L, Paquet C, Zehgnoun A, et al. Chikungunya disease outbreak, Reunion Island. Emerg Infect Dis 2006.5.

17. Cardona-Ospina JA, Henao-SanMartin V, Paniz-Mondolfi AE, et al. Mortality and fatality due to Chikungunya virus infection in Colombia. $J$ Clin Virol 2015;70:14-15.

18. Josseran J-LS L, Pierreb V, Zeghnouna A, et al. Ledrans L'impact de l'épidémie de chikungunya sur la mortalité réunionnaise. Revue d'Épidémiologie et de Santé Publique 2008;56S:S279.

19. Josseran L, Soletb J-L, Pierre V, et al. L'impact de l'épidémie de chikungunya sur la mortalité réunionnaise. Revue d'Épidémiologie et de Santé Publique 2008;56S:S279.

20. Sundararajan V, Henderson T, Perry C, et al. New ICD-10 version of the Charlson comorbidity index predicted in-hospital mortality. J Clin Epidemiol 2004;57:1288-94.

21. Chikungunya: case definitions for acute, atypical and chronic cases. Conclusions of an expert consultation, Managua, Nicaragua, 20-21 May 2015. Wkly Epidemiol Rec 2015;90:410-4

22. Kay R. Goodness of fit methods for the proportional hazards regression model: a review. Rev Epidemiol Sante Publique 1984;32(3-4):185-98.

23. Fried LP, Storer DJ, King DE, et al. Diagnosis of illness presentation in the elderly. J Am Geriatr Soc 1991;39:117-23.

24. Godaert LNF, Bartholet S, Colas S, et al. Atypical clinical presentations of acute phase Chikungunya virus infection in older patients. JAGS 2017. InPress.

25. Razmy AM. Clinical features of chikungunya infection in Sri Lanka. Asian Pac J Trop Dis 2014;4:131-4.

26. Ellis $\mathrm{G}$, Whitehead MA, Robinson D, et al. Comprehensive geriatric assessment for older adults admitted to hospital: meta-analysis of randomised controlled trials. BMJ 2011;343:d6553. 